\title{
Beamformer Design with Feedback Rate Constraints : Criteria and Constructions
}

\author{
Krishna Kiran Mukkavilli;, Ashutosh Sabharwal, Elza Erkip and Behnaam Aazhang
}

\section{Introduction}

In slow block fading channels, channel information can be obtained at the transmitter using a low delay feedback loop. In such cases, even with a few bits of channel information, feedback based transmission schemes offer substantial performance gains over channel agnostic systems with multiple transmit antennas [1,2]. In this paper, we study the design of beamformers when finite rate channel feedback is available in a multiple transmit antenna system.

In this work, we provide a geometrical framework for the analysis and design of beamformer codebooks with finite number of beamformer vectors. We present a design criterion for good beamfomer codebooks and show the equivalence of the beamformer design problem to two other known problems. First, the beamformer design problem can be directly posed as a problem of packing 2 dimensional subspaces in a $2 t$ dimensional Grassmannian manifold, $t$ being the number of transmit antennas. And second, under certain conditions, the beamformer design problem is equivalent to the construction of unitary space time constellations. Finally, our simulations demonstrate that the design criterion results in constructions which lead to near-optimal outage performance.

The paper is organized as follows. We formulate the problem in Section 2 and then proceed to analyze the outage probability of beamformers in Section 3. Motivated by the outage analysis, we present a design criterion in Section 4 . We discuss the equivalence with the subspace packing problem in Section 5 and the equivalence with the unitary space time codes is discussed in Section 6. Simulation results are given in Section 7 while we conclude in Section 8.

\section{Problem Formulation}

We consider a wireless system where the transmitter, equipped with $t$ multiple transmit antennas, communicates with a single antenna receiver. The channel fading is assumed to be quasi-static in time with the channel gains across the antenna elements being statistically independent. The $1 \times t$ channel vector is given by $\mathbf{h}=\left[h_{1}, h_{2}, . ., h_{t}\right]$, where for each $i, h_{i}$ is assumed to be circularly symmetric complex Gaussian random variable with zero mean and unit variance per complex dimension. We assume that perfect channel state information is available at the receiver. We also assume the existence of an error-free feedback channel of capacity $B$ bits/frame. The channel information at the transmitter comprises of the channel statistics and $B$ bits of quantized information about the channel reailzation available via the feedback channel. The transmitter chooses a beamforming vector from a codebook $\mathcal{C}$ based on the feedback information. Hence, corresponding to $B$ feedback bits, we have a beamformer codebook of size $N=2^{B}$, given by $\left\{C_{1}, C_{2}, . ., C_{N}\right\}$, where $C_{i} \in \mathbb{C}^{t}$ and $\left\|C_{i}\right\|_{2}=1$, for each $i$.

Without loss of generality, we let the quantizer codebook at the receiver to be the same as the beamformer codebook at the transmitter. Further, let $Q: \mathbb{C}^{t} \rightarrow \mathcal{C}$ be the channel quantizer at the receiver. Then, the beamforming vector for the channel realization $\mathbf{h}$ is given by $Q(\mathbf{h})$ and the received signal is given by

$$
y=\langle\mathbf{h}, Q(\mathbf{h})\rangle x^{*}+\eta
$$

where $x$ is the information bearing scalar and $\eta$ is complex additive white Gaussian noise which is circularly symmetric with zero mean and unit variance. Further, $\langle u, v\rangle$ is the inner product defined on $\mathbb{C}^{t}$ given by $u v^{\dagger}$, where the superscript $\dagger$ represents the conjugate transposition operation on a matrix. The average power constraint at the transmitter is given by $\mathbb{E}\left[\|x\|^{2}\right] \leq P$.

\section{Outage Analysis}

In this section, we review some results about the outage performance of beamformers from [3] for the convenience of the reader. These results will be used to motivate the design criterion for the construction of beamformers.

It is clear that the channel given in (1) is a Gaussian channel conditioned on $\mathbf{h}$ and $Q$ so that the maximum mutual information is obtained by Gaussian codebook, given by

$$
\max _{P(x \mid \mathbf{h}, Q)} I(x ; y \mid \mathbf{h}, Q)=\log _{2}\left(1+P|\langle\mathbf{h}, Q(\mathbf{h})\rangle|^{2}\right),
$$

${ }^{*}$ Corresponding author: Department of Electrical and Computer Engineering, MS 366, Rice University, Houston, Tx 77005, phone : (713)-348-2371, fax: (713)-348-6196, email: mkkiranerice.edu. A. Sabharwal and B. Aazhang are with ECE Dept., Rice University, Houston. Elza Erkip is with ECE Dept., Polytechnic University, Brooklyn. 
For each $i$, let $H_{i}^{Q} \subset \mathbb{C}^{t}$ be the set of channel realizations which will be quantized to $C_{i}$ under the quantizer $Q$. Then, the outage probability conditioned on choosing $C_{i}$ as the beamforming vector for transmission rate $R$ is given by

$$
\begin{aligned}
P_{\text {out }}\left(R, P, Q \mid C_{i}\right) & =\operatorname{Prob}\left[I(x ; y \mid \mathbf{h})<R \mid C_{i}\right]=\operatorname{Prob}\left[\log _{2}\left(1+P\left|\left\langle\mathbf{h}, C_{i}\right\rangle\right|^{2}\right)<R \mid C_{i}\right] \\
& =\operatorname{Prob}\left[\left|\left\langle\mathbf{h}, C_{i}\right\rangle\right|^{2}<\frac{2^{R}-1}{P} \mid \mathbf{h} \in H_{i}^{Q}\right] .
\end{aligned}
$$

The resulting outage probability with the codebook $\mathcal{C}$ and the quantizer $Q$ is given by

$$
P_{\text {out }}(R, P, Q)=\sum_{i=1}^{N} P\left(C_{i}\right) P_{\text {out }}\left(R, P, Q \mid H_{i}^{Q}\right) .
$$

Hence, it can be seen that the outage probability of a codebook is coupled to the quantizer at the receiver. We can prove the following lemma about the structure of the optimal quantizer $Q^{*}$ at the receiver.

Lemma 1 (Optimal Quantizer [3]) For any beamformer codebook $\mathcal{C}=\left\{C_{1}, C_{2}, \ldots C_{N}\right\}$, the outage probability is minimized by choosing for each channel realization $\mathbf{h}$, the vector $C_{i} \in \mathcal{C}$ which maximizes $\left|\left\langle\mathbf{h}, C_{i}\right\rangle\right|$.

In this section, we will use the optimal quantization rule given by $Q^{*}$ for our analysis (unless otherwise stated) and hence drop the reference to $Q^{*}$. We will now demonstrate the geometrical structure rendered to the outage evaluation problem by the optimal quantizer.

Consider the set of channel realizations which lie on the surface of the complex hypersphere given by $\|\mathbf{h}\|^{2}=\gamma$. Further, let $\mathcal{C}$ as described above be an arbitrary beamformer codebook which also serves as the quantizer codebook at the receiver. For each $i$, let $V_{i}(\gamma)$ be the Voronoi region corresponding to $C_{i}$ lying on the hypersphere $\|\mathbf{h}\|^{2}=\gamma$ given by

$$
V_{i}(\gamma)=\left\{\mathbf{h}:\|\mathbf{h}\|^{2}=\gamma,\left|\left\langle\mathbf{h}, C_{i}\right\rangle\right| \geq\left|\left\langle\mathbf{h}, C_{j}\right\rangle\right|, j \neq i\right\}
$$

Let $\Theta_{i}(\gamma) \subseteq V_{i}(\gamma)$ be the set of channel realizations corresponding to $V_{i}(\gamma)$ which do not result in outage. From (3), we know that a channel realization $\mathbf{h} \in V_{i}(\gamma)$ when used with $C_{i}$ does not result in outage if and only if $\left|\left\langle\mathbf{h}, C_{i}\right\rangle\right|^{2} \geq \gamma_{0}$ where $\gamma_{0}=\frac{2^{R}-1}{P}$. Hence, $\Theta_{i}(\gamma)$ is given by

$$
\Theta_{i}(\gamma)=\left\{\mathbf{h}: \mathbf{h} \in V_{i}(\gamma),\left|\left\langle\mathbf{h}, C_{i}\right\rangle\right|^{2} \geq \gamma_{0}\right\}
$$

We will refer to $\Theta_{i}(\gamma)$ as no-outage region in the sequel. We will use $A($.$) to denote the surface area of a region. For instance,$ $A\left(V_{i}(\gamma)\right)$ denotes the surface area of $V_{i}(\gamma)$. The surface area of the hypersphere $\|\mathbf{h}\|^{2}=\gamma$ will be denoted by $A(\gamma)$.

Note that $\mathbf{h}$ is uniformly distributed conditioned on $\|\mathbf{h}\|^{2}=\gamma$. Similarly, conditioned on $\gamma$ and $V_{i}(\gamma)$ the distribution of $\mathbf{h}$ still stays uniform. Hence the probability of no-outage with the codebook $\mathcal{C}$ conditioned on $\gamma$ can be calculated as

$$
\begin{aligned}
P_{\text {out }}^{c}\left(R, P \mid\|\mathbf{h}\|^{2}=\gamma\right) & =\sum_{i=1}^{N} P\left(C_{i} \mid\|\mathbf{h}\|^{2}=\gamma\right) P_{o u t}^{c}\left(R, P \mid C_{i},\|\mathbf{h}\|^{2}=\gamma\right) \\
& =\sum_{i=1}^{N} \frac{A\left(V_{i}(\gamma)\right)}{A(\gamma)} \frac{A\left(\Theta_{i}(\gamma)\right)}{A\left(V_{i}(\gamma)\right)}=\frac{\sum_{i=1}^{N} A\left(\Theta_{i}(\gamma)\right)}{A(\gamma)}
\end{aligned}
$$

where we have used the conditional uniform distribution of $\mathbf{h}$ to relate the fractional surface areas to the probability.

In general, it is not easy to compute the area of the no-outage region $\Theta_{i}(\gamma)$ for all $\gamma$. For this reason, we define objects with more geometrical structure known as spherical caps. These spherical caps possess enough structure to allow area computation, which in turn yields a good approximation for the area of the no-outage regions. For each $1 \leq i \leq N$ and $\gamma>0$, consider the regions $S_{i}(\gamma)$ given by

$$
S_{i}(\gamma)=\left\{\mathbf{h}:\|\mathbf{h}\|^{2}=\gamma,\left|\left\langle\mathbf{h}, C_{i}\right\rangle\right|^{2} \geq \gamma_{0}\right\} .
$$

$S_{i}(\gamma)$ is formed by the intersection of the hypersphere $\|\mathbf{h}\|^{2}$ with an hyperplane. Such regions resemble a spherical cap in the the case of $\mathbb{R}^{3}$ and hence the name for the general case also. While no-outage regions are non-overlapping for all $\gamma$, the spherical caps do overlap for large $\gamma$. However, it is interesting to note the following relationship between the two.

Lemma 2 [3] For all $\gamma>0, \bigcup_{i=1}^{N} \Theta_{i}(\gamma)=\bigcup_{i=1}^{N} S_{i}(\gamma)$.

Further, since $\Theta_{i}(\gamma)$ are non-overlapping for all $\gamma$, we have using Lemma 2,

$$
\sum_{i=1}^{N} A\left(\Theta_{i}(\gamma)\right)=A\left(\bigcup_{i=1}^{N} \Theta_{i}(\gamma)\right)=A\left(\bigcup_{i=1}^{N} S_{i}(\gamma)\right)
$$


Hence, equation (8), i.e., the probability of successful transmission, for a given channel norm, can also written as

$$
P_{\text {out }}^{c}\left(R, P \mid\|\mathbf{h}\|^{2}=\gamma\right)=\frac{\sum_{i=1}^{N} A\left(\Theta_{i}(\gamma)\right)}{A(\gamma)}=\frac{A\left(\bigcup_{i=1}^{N} S_{i}(\gamma)\right)}{A(\gamma)} .
$$

We will now relate $A\left(\bigcup_{i=1}^{N} S_{i}(\gamma)\right)$ to the area of individual spherical caps, $A\left(S_{i}(\gamma)\right)$, for different values of $\gamma$. It can be shown that $S_{i}(\gamma)$ are all congruent for different $i$ so that $A\left(S_{i}(\gamma)\right)$ is the same for all $i$. Also, $A\left(S_{i}(\gamma)\right)$ is given by [3]

$$
A\left(S_{i}(\gamma)\right)=\left\{\begin{array}{cc}
0, & \gamma<\gamma_{0} \\
\frac{2 \pi^{t} \sqrt{\gamma}\left(\gamma-\gamma_{0}\right)^{t-1}}{(t-1) !}, & \gamma \geq \gamma_{0}
\end{array}\right.
$$

Further, the area of the $t$-dimensional complex hypersphere of radius $\sqrt{\gamma}$ is given by [3]

$$
A(\gamma)=\frac{2 \pi^{t}(\sqrt{\gamma})^{(2 t-1)}}{(t-1) !} .
$$

Note that for small values of $\gamma$, the spherical caps $S_{i}(\gamma)$ are non-overlapping while for larger values of $\gamma$, some of the spherical caps do overlap. We define the overlap radius of a codebook $\mathcal{C}$ as the channel norm squared at which this transition takes place.

Definition $3:$ For any beamformer codebook $\mathcal{C}$, the overlap radius is defined as the largest channel norm squared for which no two spherical caps overlap. It is denoted by $\gamma^{*}(\mathcal{C})$.

We will now relate the overlap radius of $\mathcal{C}$ to its outage probability and argue that the outage performance of a codebook can be improved by increasing its overlap radius. When $\gamma<\gamma^{*}$, the spherical caps form disjoint sets so that we have

$$
A\left(\bigcup_{i=1}^{N} S_{i}(\gamma)\right)=\sum_{i=1}^{N} A\left(S_{i}(\gamma)\right)=N\left(\frac{2 \pi^{t} \sqrt{\gamma}\left(\gamma-\gamma_{0}\right)^{t-1}}{(t-1) !}\right), \gamma_{0} \leq \gamma<\gamma^{*} .
$$

On the other hand, when $\gamma \geq \gamma^{*}$, the spherical caps are no longer disjoint and hence we have

$$
A\left(\bigcup_{i=1}^{N} S_{i}(\gamma)\right) \leq \sum_{i=1}^{N} A\left(S_{i}(\gamma)\right)=N\left(\frac{2 \pi^{t} \sqrt{\gamma}\left(\gamma-\gamma_{0}\right)^{t-1}}{(t-1) !}\right), \gamma \geq \gamma^{*} .
$$

Substituting (13) and the above set of relations in (11), we get

$$
\begin{aligned}
P_{\text {out }}^{c}\left(R, P \mid\|\mathbf{h}\|^{2}=\gamma\right) & =N\left(1-\frac{\gamma_{0}}{\gamma}\right)^{(t-1)}, \gamma<\gamma^{*} \\
& \leq \min \left(1, N\left(1-\frac{\gamma_{0}}{\gamma}\right)^{(t-1)}\right), \gamma^{*}<\gamma
\end{aligned}
$$

From (16) we observe that for a larger overlap radius $\gamma^{*}$, the spherical caps do not overlap upto a larger channel norm thus contributing more surface area to the no-outage regions (correspondingly, the equality in (16) holds upto a large channel norm). On the other hand, the contribution of the spherical caps to the no-outage regions is reduced when the overlap radius is small thus allowing the inequality in (16) to set in for a smaller channel norm. Hence, it is clear that to improve the performance of the codebook $\mathcal{C}$, the overlap radius should be made larger thus giving the design criterion

$$
\max _{\{\mathcal{C}\}} \gamma^{*}(\mathcal{C})
$$

We will relate the overlap radius of the codebook to its constituent beamforming vectors in the next section. To conclude the section, we point out that (16) can be integrated over the distribution of the channel to result in the following universal lower bound on the outage probability of a beamformer codebook consisting of a finite number of vectors. In particular we have,

$$
P_{\text {out }}(R, P) \geq 1-N e^{-\gamma_{0}}+e^{-\gamma_{1}} \sum_{k=0}^{t-1}\left(\frac{N\left(\gamma_{1}-\gamma_{0}\right)^{k}-\gamma_{1}^{k}}{k !}\right)
$$

where

$$
\gamma_{0}=\frac{2^{R}-1}{P} \text { and } \gamma_{1}=\frac{\gamma_{0}}{\left(1-\left(\frac{1}{N}\right)^{\left(\frac{1}{t-1}\right)}\right)}
$$




\section{Design Criterion}

In this section, we will relate the design criterion for good beamformer codeboks given in (17) to the constituent vectors of the beamformer codebook. The calculation of the overlap radius $\gamma^{*}$ for a codebook is shown in the following lemma.

Lemma 4 (Overlap radius) For any beamformer codebook $\mathcal{C}$, we have

$$
\gamma^{*}(\mathcal{C})=\left(\frac{2 \gamma_{0}}{1+\max _{i, j: i \neq j}\left|\left\langle C_{i}, C_{j}\right\rangle\right|}\right)
$$

Proof Let $\mathcal{C}=\left\{C_{1}, C_{2}, . . C_{N}\right\}$ be any beamformer comprising of $N$ beamforming vectors, with $C_{i} \in \mathbb{C}^{t}$ for each $i$. Following the notation developed earlier, for a given channel norm $\sqrt{\gamma}$, let $V_{i}(\gamma)$ and $\Theta_{i}(\gamma)$ be the Voronoi region and no-outage region corresponding to $C_{i}$, for each $i$. Further, let $S_{i}(\gamma)$ be the spherical cap around $C_{i}$ as described earlier. Let $\sqrt{\gamma_{i j}}$ be the smallest channel norm for which $S_{i}(\gamma)$ and $S_{j}(\gamma)$ intersect. Then, it is clear that $\gamma^{*}$ can be calculated as $\gamma^{*}=\min _{1 \leq i<j \leq N} \gamma_{i j}$. Hence, we will first evaluate $\gamma_{i j}$ for a given $i$ and $j$ and then calculate $\gamma^{*}$.

Consider the smallest channel norm $\sqrt{\gamma_{12}}$ at which $S_{1}(\gamma)$ intersects $S_{2}(\gamma)$. By the definition of $S_{i}(\gamma)$, if $\mathbf{h}$ is a vector which lies on the boundary of $S_{i}(\gamma)$, then $\mathbf{h}$ satisfies $\left|\left\langle\mathbf{h}, C_{i}\right\rangle\right|^{2}=\gamma_{0}$. Let $\mathbf{h}$ be a vector which lies on the boundary of $S_{1}(\gamma)$ and $S_{2}(\gamma)$. Also, let $\|\mathbf{h}\|^{2}=\gamma$. Then, $\gamma_{12}$ is the smallest value of $\gamma$ which satisfies the following conditions.

$$
\begin{aligned}
\mathbf{h} & =\sqrt{\gamma}\left(\alpha C_{1}+\beta C_{2}+\delta C\right) \\
\|\mathbf{h}\|_{2}^{2} & =\gamma \\
\left|\left\langle h, C_{1}\right\rangle\right|^{2} & =\gamma_{0} \\
\left|\left\langle h, C_{2}\right\rangle\right|^{2} & =\gamma_{0}
\end{aligned}
$$

where $C \in \mathbb{C}^{t}$ is a unit vector orthogonal to both $C_{1}$ and $C_{2}$. Also, $\alpha, \beta$ and $\gamma$ are complex scalars.

On equating the left hand side of (23) and (24), we get

$$
\begin{aligned}
|\alpha|^{2}+|\beta|^{2}\left|\left\langle C_{1}, C_{2}\right\rangle\right|^{2} & =|\beta|^{2}+|\alpha|^{2}\left|\left\langle C_{1}, C_{2}\right\rangle\right|^{2} \\
\Rightarrow|\alpha| & =|\beta| .
\end{aligned}
$$

(where we have ignored the less interesting case of $\left|\left\langle C_{1}, C_{2}\right\rangle\right|=1$ which happens when $C_{1}$ is a scalar multiple of $C_{2}$. In this case, we can drop one of the two vectors from $\mathcal{C}$ without affecting the outage performance of $\mathcal{C}$.)

Without loss of generality, let $\alpha$ be real and $\beta=\alpha e^{j \theta}$. Substituting this in (23), we get

$$
\gamma_{0}=\gamma \alpha^{2}\left(1+\left|\left\langle C_{1}, C_{2}\right\rangle\right|^{2}+2 \operatorname{Re}\left(e^{-j \theta}\left\langle C_{1}, C_{2}\right\rangle\right)\right) .
$$

Similarly, substituting for $\alpha$ and $\beta$ in (21), we get

$$
2 \alpha^{2}\left(1+\operatorname{Re}\left(e^{-j \theta}\left\langle C_{1}, C_{2}\right\rangle\right)\right)+|\delta|^{2}=1
$$

Now, substituting the value of $\alpha$ from (27) in (26) and simplifying, we get

$$
\gamma=\frac{2 \gamma_{0}\left(1+\operatorname{Re}\left(e^{-j \theta}\left\langle C_{1}, C_{2}\right\rangle\right)\right)}{\left(1-|\delta|^{2}\right)\left(1+\left|\left\langle C_{1}, C_{2}\right\rangle\right|^{2}+2 \operatorname{Re}\left(e^{-j \theta}\left\langle C_{1}, C_{2}\right\rangle\right)\right)}
$$

Finally, note that $\gamma_{12}$ is the minimum value of $\gamma$ which satisfies this equation. To evaluate $\gamma_{12}$, we minimize $\gamma$ with respect to $\theta$ and $\delta$. Clearly, $\gamma$ is minimized when $|\delta|=0$ (i.e., the spherical caps $S_{1}(\gamma)$ and $S_{1}(\gamma)$ first intersect in the subspace formed by $C_{1}$ and $C_{2}$ ). Let $\gamma$ assume its minimum value at $\theta=\theta_{12}$. Differentiating $\gamma$ with respect to $\theta$ and setting it to zero at $\theta=\theta_{12}$, we get

$$
\begin{aligned}
\operatorname{Re}\left(-j e^{-j \theta_{12}}\left\langle C_{1}, C_{2}\right\rangle\right) & =0 \\
\Rightarrow \phi-\theta_{12} & =k \pi
\end{aligned}
$$

where $\phi=$ phase $\left(\left\langle C_{1}, C_{2}\right\rangle\right)$ and $k$ is any integer.

Evaluating the second derivative and setting it greater than zero at $\theta_{12}$ (for a minima at $\theta_{12}$ ), we get

$$
\operatorname{Re}\left(e^{-j \theta_{12}}\left\langle C_{1}, C_{2}\right\rangle\right)>0
$$

From (29) and (30), we see that $\gamma$ is minimized at $\theta_{12}=\phi+2 k \pi$, for any integer $k$. Set $k=0$ to give $\theta_{12}=\phi$. Substituting this value of $\theta_{12}$ in (28), we get

$$
\gamma_{12}=\frac{2 \gamma_{0}}{1+\left|\left\langle C_{1}, C_{2}\right\rangle\right|}
$$


Finally, $\gamma^{*}(\mathcal{C})$ is given by

$$
\begin{aligned}
\gamma^{*}(\mathcal{C}) & =\min _{i, j: i \neq j} \gamma_{i j} \\
& =\left(\frac{2 \gamma_{0}}{1+\max _{i, j: i \neq j}\left|\left\langle C_{i}, C_{j}\right\rangle\right|}\right) .
\end{aligned}
$$

Hence, the overlap radius is a function of the two closest vectors in the beamformer. The design criterion for beamformers $\max _{\{\mathcal{C}\}} \gamma^{*}(\mathcal{C})$, given in (17), is now equivalently given by the following lemma.

Lemma 5 [Design criterion] The design criterion (17) for a codebook $\mathcal{C}$ comprising of $N$ beamforming vectors for $t$ transmit antennas is equivalently by

$$
\min _{\mathcal{c} \in \underbrace{\min ^{t} \times \cdots \times \mathbb{C}^{t}}_{N}} \max _{\substack{i, j: \vdots \\ 1 \leq i \neq j \leq N}}\left|\left\langle C_{i}, C_{j}\right\rangle\right|
$$

The design criterion essentially seeks maximal 'separation' for the two closest vectors in the beamformer codebook. This is similar to the design criterion of signal constellations which maximizes the minimum Euclidean distance between any two points in the signal constellations. Further discussions on the design criterion can be found in [3]. However, it is worthwhile to note that the design criterion results in the well known selection diversity for the special case of $N=t$ as expected.

Corollary 6 When $N=t$, the design criterion in (33) leads to selection diversity with $\gamma^{*}=2 \gamma_{0}$.

\section{Packings in Grassmannian Spaces}

It is interesting to note that the design criterion presented in the previous section can be posed as a problem of packing subspaces in Grassmannian spaces, which was discussed in detail in [4]. The similarity could potentially lead to new construction methods for beamformer codebooks. Consider the vector $C_{i} \in \mathbb{C}^{t}$. We can write $C_{i}=C_{i R}+\jmath C_{i I}$, where $C_{i R}$ and $C_{i I}$ are the real and the imaginary parts of $C_{i}$ respectively. Now consider the two dimensional subspace, $F_{i}$, of $\mathbb{R}^{2 t}$ formed by the span of the columns of the matrix $M_{i}$ given by

$$
M_{i}=\left[\begin{array}{cc}
C_{i R} & C_{i I} \\
C_{i I} & -C_{i R}
\end{array}\right]
$$

A measure of distance, called the chordal distance, between the subspaces $F_{i}$ and $F_{j}$ is given by,

$$
d_{c}\left(F_{i}, F_{j}\right)=\sqrt{\sin ^{2}\left(\theta_{1}\right)+\sin ^{2}\left(\theta_{2}\right)},
$$

where $\theta_{1}$ and $\theta_{2}$ are the principal angles between $F_{i}$ and $F_{j}$ [5]. It can be shown that $\left|\left\langle C_{i}, C_{j}\right\rangle\right|^{2}=1-\frac{d_{c}^{2}\left(F_{i}, F_{j}\right)}{2}$, [3] so that the design criterion in (33) can be restated as

$$
\max _{\{F\}} \min _{1 \leq i<j \leq N} d_{c}^{2}\left(F_{i}, F_{j}\right),
$$

where $F$ is a set of cardinality $N$, consisting of 2 dimensional subspaces of $\mathbb{R}^{2 t}$ formed by matrices of the nature given by (34). This is the packing problem discussed in [4].

\section{Unitary Space Time Codes as Beamformers}

One of the interesting properties of the design criterion presented in Lemma 5 is that it relates the finite size beamformer codebook design to the design of good unitary space time codes for non-coherent communcations. Unitary space time constellations for multiple antenna systems were introduced by Marzetta and Hochwald in [6]. In particular, it was shown that unitary space time constellations achieve the capacity of multiple antenna systems when the channel information is not available at either the transmitter or the receiver. The design criterion for unitary space time constellations to minimize the pair-wise error probability was given in [7]. A unitary space time constellation consists of signals $\Phi_{1}, \Phi_{2}, . . \Phi_{N}$, where $\Phi_{i} \in \mathbb{C}^{T \times M}$, where $T$ is the block length of the code (less than or equal to the coherence time of the channel), $M$ is the number of transmit antennas and $N$ is the number of codewords in the constellation. Also, the unitarity condition implies that $\Phi_{i}^{\dagger} \Phi_{i}=I$ for each $i$. It was shown that the design criterion for unitary space time constellations, which minimize pair-wise error probability, is to minimize $\delta$, where $\delta$ is defined as

$$
\delta=\max _{1 \leq l<l^{\prime} \leq N}\left\|\Phi_{l}^{\dagger} \Phi_{l^{\prime}}\right\|
$$

where the norm used above is a scaled Frobenius norm of a matrix, the scaling factor being given by $M$ in this case. 


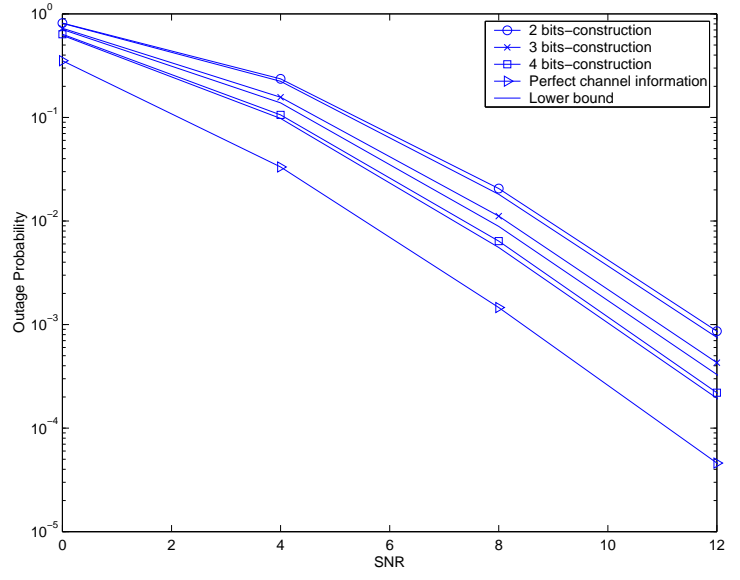

(a) Construction from computer based search

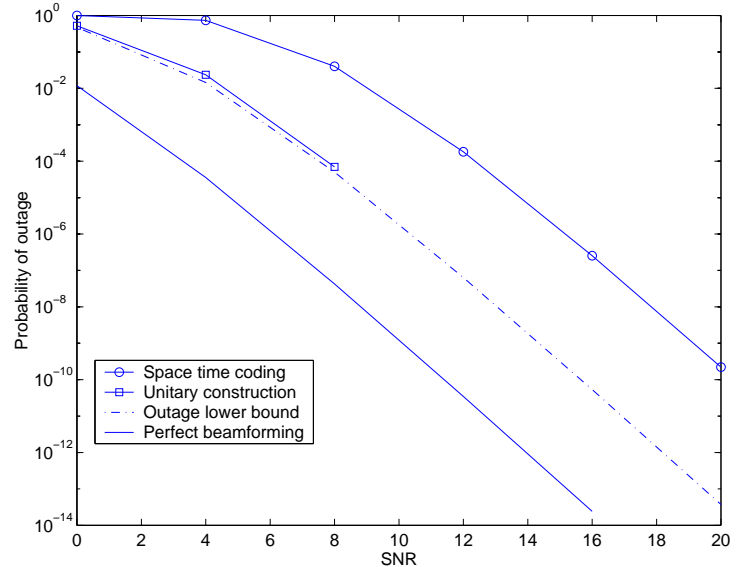

(b) Systematic unitary space time constellation

Figure 1: Performance of beamformers for $R=2 \mathrm{bits} / \mathrm{sec} / \mathrm{Hz}$ : (a) Construction for 4 transmit antennas, and (b) Unitary space time code as beamformer for 8 transmit antennas

Recall that, for the finite feedback problem considered in this paper, if the beamformer codebook $\mathcal{C}$ comprises of $\left\{C_{1}, C_{2}, . ., C_{N}\right\}$, then the design criterion for good beamformers is

$$
\min _{\mathcal{C} \in \mathbb{C}^{t}} \max _{1 \leq l<l^{\prime} \leq N}\left|\left\langle C_{l}, C_{l^{\prime}}\right\rangle\right|
$$

where $\left\langle C_{l}, C_{l^{\prime}}\right\rangle=C_{l} C_{l^{\prime}}^{\dagger}$. The criterion (37) is equivalent to the criterion for beamformers given in (38), if we set $T=t$ and $M=1$ in (37). We see an equivalence between coherence time, in the unitary constellation design problem, and the number of transmit antennas, in the beamforming design problem. Hence, the design of beamformers is equivalent to the problem of the design of unitary space time constellations under these conditions.

\section{Simulation Results}

The performance results of the beamformers cosntructed using the design criterion of Lemma 5 for the case of 4 transmit antennas with a single receive antenna is given in Figure 1(a). We have presented the case of 2,3 and 4 bits of feedback for the case of $R=2 \mathrm{bits} / \mathrm{sec} / \mathrm{Hz}$. Computer search was used to generate the codebooks. The lower bound on outage probability from (18) is used for reference in the simulations. The outage probability in all the cases is very close to the lower bound thus reflecting the tightness of the lower bound as well as the near-optimality of the constructions. The performance of a unitary space time code as a beamformer for 8 transmit antennas with $R=2 \mathrm{bits} / \mathrm{sec} / \mathrm{Hz}$ is shown in Figure 1(b). We have used the systematic construction from [7] corresponding to $M=1, T=8$ and $N=16$. It can be seen that the performance of the unitary space time code is less than $0.05 d B$ away from the lower bound, thus demonstrating its excellent performance.

\section{Conclusions}

In this paper, we have presented a design criterion for constructing good beamformer codebooks when quantized channel feedback is available at the transmitter. We also demonstrated the equivalence of the beamformer design with several other interesting problems known in the literature.

\section{References}

[1] A. Narula, M.J. Lopez, M.D. Trott, and G.W. Wornell, "Efficient use of side information in multiple-antenna data transmission over fading channels," IEEE Journal on selected areas in communications, pp. 1423-1436, October 1998.

[2] S. Bhashyam, A. Sabharwal, and B. Aazhang, "Feedback gain in multiple antenna systems," IEEE trans. on communications, vol. 50, pp. 785-798, May 2002.

[3] K. K. Mukkavilli, A. Sabharwal, E. Erkip and B.Aazhang, "On Beamforming with Finite Rate Feedback in Multiple Antenna Systems," submitted to IEEE trans. on Information Theory, October 2002.

[4] J. H. Conway, R. H. Hardin and N. J. A. Sloane, "Packing lines, planes, etc., packings in grassmannian spaces," Experimental Mathematics, vol. 5, pp. 139-159, 1996.

[5] G. H. Golub and C. F. Van Loan, Matrix Computations, The Johns Hopkins University Press, Baltimore, 1983.

[6] T.L. Marzetta and B.M. Hochwald, "Capacity of a mobile multiple-antenna communication link in rayleigh flat fading," IEEE transactions on Information Theory, pp. 139-157, January 1999.

[7] B.M. Hochwald and T.L. Marzetta, "Unitary space-time modulation for multiple-antenna communications in rayleigh flat fading," IEEE transactions on Information Theory, vol. 46, pp. 543-564, March 2000. 